\title{
Communication \\ Micro-Range Actuation by Pressure-Induced Elastic Deformation of 316L Steel Membranes Produced by Laser Powder Bed Fusion
}

\author{
Florian Fettweis ${ }^{1, *}\left(\mathbb{C}\right.$, Bjorn Verrelst $^{2}$ and Svend Bram ${ }^{1}(\mathbb{C}$ \\ 1 Thermo and Fluid Dynamics (FLOW), Vrije Universiteit Brussel (VUB), 1050 Brussels, Belgium; \\ svend.bram@vub.be \\ 2 Applied Mechanics (MECH), Vrije Universiteit Brussel (VUB), 1050 Brussels, Belgium; bjorn.verrelst@vub.be \\ * Correspondence: Florian.Fettweis@vub.be
}

Citation: Fettweis, F.; Verrelst, B.; Bram, S. Micro-Range Actuation by Pressure-Induced Elastic Deformation of 316L Steel Membranes Produced by Laser Powder Bed Fusion. Actuators 2021, 10, 296. https:// doi.org/10.3390/act10110296

Academic Editor: Richard Yongqing $\mathrm{Fu}$

Received: 4 October 2021

Accepted: 3 November 2021

Published: 6 November 2021

Publisher's Note: MDPI stays neutral with regard to jurisdictional claims in published maps and institutional affiliations.

Copyright: (c) 2021 by the authors. Licensee MDPI, Basel, Switzerland. This article is an open access article distributed under the terms and conditions of the Creative Commons Attribution (CC BY) license (https:/ / creativecommons.org/licenses/by/ $4.0 /)$.

\begin{abstract}
In this paper, fundamental research is performed on membrane type actuators made out of 316L stainless steel, manufactured with Laser powder bed fusion (LPBF). A total of six membranes with membrane thicknesses ranging from $0.6 \mathrm{~mm}$ up to $1.2 \mathrm{~mm}$ were scanned using a high precision metrology system to measure the membrane for displacement at different actuating pressures. The membranes were furthermore investigated for roughness, porosity and thickness. This showed that the thinnest membranes skewed in the print direction when actuated. The remaining membranes achieved higher specific displacements than finite element simulations (FES) predicted, due to surface roughness and porosity. Membrane type actuators can be used for precise actuation within the micrometre range. LPBF allows the creation of internal pockets and membranes in a single metal piece. In opposition to the more commonly used polymers for membrane-type actuators, LPBF steel printed parts offer high stiffness and actuation force. However, due to limitations of the LPBF process on thin walls, large deviations from FES occur. In this paper, a CAD and FES compensation strategy is suggested, which makes future, more complex and effective, designs possible.
\end{abstract}

Keywords: additive manufacturing application; laser powder bed fusion; micro-range actuation; membrane actuator; 316L stainless steel

\section{Introduction}

Accurate micro-positioning of a heavy load remains challenging. Typically XY-crosstables can be used for precise micro-positioning; however, their maximal load is limited up to about several $10 \mathrm{~kg}$. Even specialised heavy duty XY-cross-tables struggle to carry loads over 200 to $300 \mathrm{~kg}$ and only achieve so while increasing the size and weight of the table to a hulking presence. In addition to the challenge of micro-actuating heavy static loads, dynamic loading of XY-tables is not possible. Ideally a micro-positioning device would be backlash free and extremely stiff while actuating and while holding. The stiffness allows for a high static load with minimal deflection. However, there is also the possibility for high dynamic loads with a neglect-able amplitude.

A methods for achieving a high power density in a compact actuator is the usage of an external pressure source. In classic hydraulic actuators, a pistons with hydraulic seals is displaced under pressure, which can achieve a high load and stiffness. However, friction and flexing of the hydraulic seals result in backlash and inaccuracy of the actuator. More importantly, this type of actuator delivers a constant force (depending on the applied pressure), not a specific displacement. Alternatively, hydraulic actuation can be achieved by deforming an internal pocket in an actuator. Similar to a balloon, these internal pockets can be pressurised to deform the geometry. This intentional elastic deformation of the geometry can be used as an actuator with a high actuation force, and a relatively small footprint. Nevertheless, the stroke is determined by the yield of the material, thus choosing a stiff material, this limits the maximal stroke. 
Actuators using intentional elastic deformation or flexibility are already successfully manufactured with polymers and plastics using classic techniques such as casting and moulding techniques [1,2], but also additive manufacturing techniques such as microstereolithography [3], fused layer manufacturing [4,5] and selective laser sintering [6]. These actuators achieve high precision and accuracy. However, actuators made out of polymers are limited by the material properties of the polymers, and thus have relatively low stiffness and actuation force [7]. In order to actuate heavy loads, the high actuation force is crucial, whereas the low stiffness of these actuators result in large dynamic responses on an excitation's. Because of the simplicity, ease of use and precision of membrane-type actuators, a different material and manufacturing technique is investigated.

Additive manufacturing allows for the manufacturing of complex thin-walled structures with internal hollow pockets. One of the major advantages of additive manufacturing over conventional machining for this kind of actuator is the possibility to create the internal pockets in a single component. For a hydraulic or pneumatic positioner, this is especially interesting, as there is no need for hydraulic seals and/or tolerated fittings between moving parts. This increases the ease of use and reduces the chance of leakage of the power transmitting fluid. In this communication laser powder bed fusion of 316 stainless steel is evaluated for the manufacturing of membrane type actuators. 316L stainless steel has high strength values (Ultimate tensile strength up to $700 \mathrm{GPa}$ and Yield strength up to $550 \mathrm{MPa}$ ) and a high Young's modulus around $200 \mathrm{GPa}$, which results in high stiffness and a high actuation force [8-10]. However, as the combination of this technique with 316 stainless steel membrane type actuators is not yet used, as of the knowledge of the writers, thus fundamental research is required.

In addition to the importance of the material properties, the geometry itself also plays a crucial role in the maximum displacement (stroke), required pressure, actuation force and stiffness. The myriad of geometries that can be used as a positioning device using intentional elastic deformation makes an effective and predictable design without fundamental insights impossible. Therefore, the research in this paper will focus on a basic round membrane design which will lay the groundwork for future more complex designs. The basic shape allows a more in-depth investigation of one of the key features in any compliant pressurised actuator's geometry, namely the thickness of the membrane between the pressurised zone and the outside world. A variety of membrane thicknesses between $0.6 \mathrm{~mm}$ and $1.2 \mathrm{~mm}$ will be investigated on specific displacement $\left(\frac{d D}{d P}\right)$, skewing (using coordinate measuring scanning machines) and effective wall thickness (using microscopic imaging) to evaluate finite element simulations. Ultimately, the results found in this research will be used to set up a basis consisting of guidelines and design rules for novel, high stiffness, compact micro positioning devices manufactured using LPBF, based on intentional elastic deformation.

\section{Materials and Methods}

\subsection{Design}

The prototype design is based on a membrane type positioner [7] as shown in Figure 1. The centre of the membrane is thickened to achieve a uniform flat expansion surface to investigate skewing during actuation. The prototype positioner has two tapped holes, one for filling and feeding, while one serves as a bleeding hole for venting. The outer and inner radius of the membrane are, respectively, $26.0 \mathrm{~mm}$ and $14.5 \mathrm{~mm}$. The thickened part has a thickness of $2.9 \mathrm{~mm}$. Each prototype consists of two parallel membranes to reduce the number of parts to be printed. Creating thin walls/membranes using LPBF is still challenging, as the typical tolerance of the LPBF process is around $0.1 \mathrm{~mm}$ [11]. On a membrane, this could result in a worst-case scenario, in a $0.2 \mathrm{~mm}$ thinner membrane than designed. Furthermore is the print direction of utmost importance. During the printing process, the membrane has to transfer generated heat required for melting the metal (and not warp during or after the process). Because of these limiting factors, a minimum membrane thickness of $0.6 \mathrm{~mm}$ is chosen. Membranes thicker than $1.2 \mathrm{~mm}$ resulted in low 
expansion rates in simulations and overall low strokes, and therefore, $1.2 \mathrm{~mm}$ is chosen as a maximum thickness. In order to assess the influence of the thickness, three prototypes are created, each with two different membrane thicknesses. Prototype one has membrane thicknesses of $1.0 \mathrm{~mm}$ and $1.2 \mathrm{~mm}$, prototypes two and three have membrane thicknesses of $0.6 \mathrm{~mm}$ and $0.8 \mathrm{~mm}$. Prototypes two and three are identical in design to assess repeatability. An aluminium housing allows the testing of a single membrane, without actuating the other membrane. A drawing of the cross-section of the test setup can be seen in Figure 2.
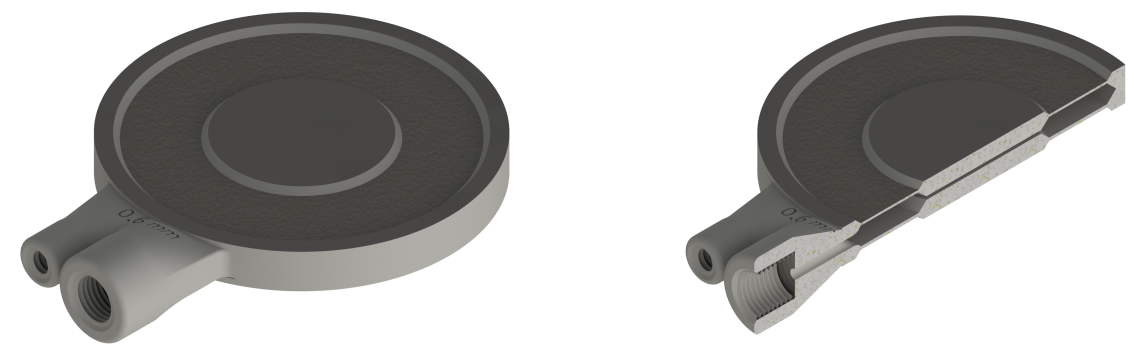

Figure 1. Render of the prototype (left pane) with designed membrane thicknesses $0.6 \mathrm{~mm}$ and $0.8 \mathrm{~mm}$ and a cross-section image (right pane). In the middle of the membrane the thickened part can be seen. The larger of the two threaded connections is used as feeding hole, the smaller one is used as venting hole.

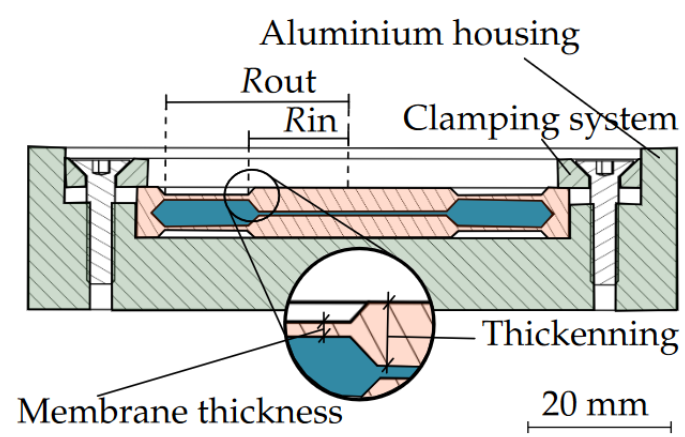

Figure 2. Schematic drawing of the membrane actuator in test setup. $R_{o u t}$, the outer radius of the membrane, is $26.0 \mathrm{~mm}$. $R_{i n}$, the inner radius of the membrane and the radius of the thickened part, is $14.5 \mathrm{~mm}$. The thickening is $2.9 \mathrm{~mm}$ thick, whereas the membrane thickness ranges between $0.6 \mathrm{~mm}$, $0.8 \mathrm{~mm}, 1.0 \mathrm{~mm}$ up to $1.2 \mathrm{~mm}$. An aluminium ring clamps the actuator on the housing. The housing has a radius of $4.5 \mathrm{~mm}$ and height of $22.5 \mathrm{~mm}$. The clamping allows the expansion of the upper membrane and suppresses the expansion of the lower membrane.

\subsection{Simulation}

The expansion ratio and stroke are simulated using the finite element simulation (FES) Stress Analysis of Autodesk Inventor. The following mesh settings are used; a bounding box of $60 \times 60 \times 7 \mathrm{~mm}$; an average element size of 0.01 times the bounding box length with a minimum of 0.005 times the bounding box length: a grading factor of 1.5; a max turn angle of $60^{\circ}$. A local smaller mesh is used for the thin part of the membrane of $0.1 \mathrm{~mm}$. A convergence analysis is performed, which refined the meshing until a maximum of $1 \%$ divergence in Von Mises stress is simulated. Thickness between $0.3 \mathrm{~mm}$ and $1.4 \mathrm{~mm}$ with a step of $0.05 \mathrm{~mm}$ were used to simulated the specific displacement at a pressure of $1 \mathrm{bar}$ on the membrane. The model simulates elastically (even when yield stresses are achieved), thus the simulated specific displacement is independent of the applied pressure. For future use in this communication, all simulations are connected as a continuous line.

Young's modulus and Yield strengths are given by the supplier and are, respectively, $200 \mathrm{GPa}$ and $500 \mathrm{MPa}$, with a Poisson's ratio of 0.3. Although the mechanical properties of LPBF manufactured parts are an-isotropic, the prototypes are simulated isotropically. The error made by this simplification is expected to be less than $10 \%$ as the most important 
parameters (Yield strength for maximum pressure and Young's modulus for displacement ratio) differ less than $10 \%$ in orthogonal directions. In order to minimise porosity, all prototypes were printed in the same batch, with optimised printing parameters.

Table 1. Simulated specific displacements, maximum pressure and stroke for each prototype (number) and membrane (letter). Thicker membranes can withstand higher pressures before yield, but thinner membranes achieve a higher maximum stroke.

\begin{tabular}{|c|c|c|c|c|c|}
\hline \multirow{2}{*}{ Property } & \multirow{2}{*}{ Unit } & \multicolumn{4}{|c|}{ Prototype } \\
\hline & & 1A & 1B & $2 \mathrm{~A} \& 3 \mathrm{~A}$ & $2 B \& 3 B$ \\
\hline Design thickness & $\mathrm{mm}$ & 1.20 & 1.00 & 0.80 & 0.60 \\
\hline Simulated specific displacement & $\mu \mathrm{m}$ bar $^{-1}$ & 6.78 & 10.57 & 18.47 & 39.10 \\
\hline Simulated maximum pressure & bar & 17.45 & 11.10 & 8.69 & 5.34 \\
\hline Simulated stroke ${ }^{a}$ & $\mu \mathrm{m}$ & 118.26 & 117.34 & 160.70 & 208.73 \\
\hline
\end{tabular}

In general, thinner membranes result in higher specific displacement $\left(\left[\mu \mathrm{mbar}{ }^{-1}\right]\right)$ and stroke $([\mu \mathrm{m}])$ in the FEM simulations (see Table 1$)$. The maximum pressure and stroke are dependent on the load on the membrane, whereas the expansion ratio only depends on the membrane properties. A load would push the membrane in the opposite direction of the actuation and thus decreases stresses in the membrane (within certain limits). Simulations predict an equivalent force generated by the applied pressure of $224 \mathrm{~N} \mathrm{bar}^{-1}$ to $235 \mathrm{~N} \mathrm{bar}^{-1}$ (resp. for membrane thicknesses $1.2 \mathrm{~mm}$ and $0.6 \mathrm{~mm}$ ), thus a force of $235 \mathrm{~N}$ could be counteracted by a pressure of 1 bar without displacement of the thickened central part of the membrane. In Table 1, the simulated maximum pressure and stroke before yielding occurs and without load are depicted.

\subsection{Scan}

The prototypes displacements are scanned using a Nikon HN-C3030 3D metrology system with a linear resolution of $20 \mu \mathrm{m}$ (see Figure $3 a, b$ ). The scans are validated using a high precision coordinate measurement machine (CMM) (see Figure 3c). The Nikon HN-C3030 3D metrology system proved to be reliable and precise for this application, with the possibility to investigate the whole membrane surface in detail and as a result, is chosen over the CMM machine.

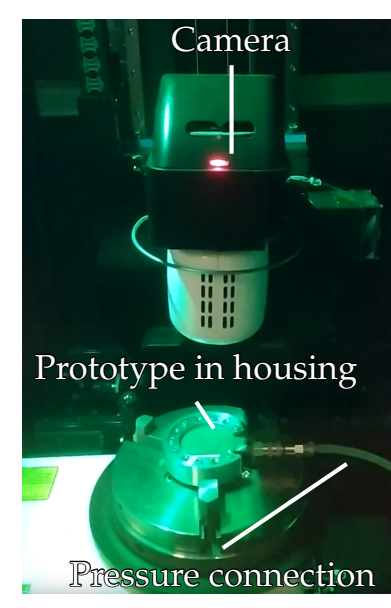

(a)

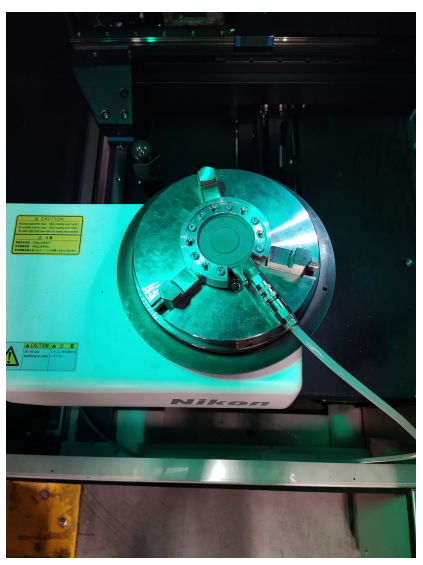

(b)

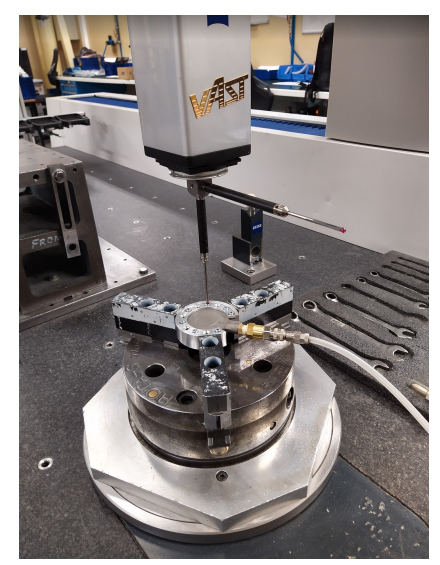

(c)

Figure 3. Pictures of the actuator in the Nikon HN-C3030 scanning system and Zeiss CMM machine. (a) Front view of the prototype positioned in the Nikon HN-C3030 metrology scanning system. (b) Top view of the prototype positioned in the Nikon HN-C3030 metrology scanning system. (c) Side view of the prototype positioned in the Zeiss CMM machine. 
Each actuator is scanned before pressure is applied and subsequently scanned at several incremental pressures (with a minimum pressure step of 0.5 bar) up to a maximum pressure, which is lower than the maximum simulated pressure (see Table 1). The maximum pressures in the actuators are kept low to ensure operation in the elastic domain. The safety factor ( $\left.\mathrm{SF}=\frac{\sigma_{\text {simulated }}}{\sigma_{\text {yild }}}\right)$ is always chosen higher than $\mathrm{SF}=2$, to compensate for the manufacturing accuracy of the LPBF process (being approximately $0.1 \mathrm{~mm}$ ) and to avoid yield of the membranes.

The point cloud measured before applying pressure is used to calculate a reference mesh, each subsequent scan (with a pressurised membrane) is then compared to the reference mesh to determine the displacement ( $\mathrm{D}$ in micrometre). The aim of the study is to examine the elastic properties of the membranes. Operation within the elastic domain is ensured by another scan without pressure, which is compared with the reference mesh.

First, the mean displacement of $1 \mathrm{~mm}$ wide cross sections, through the centre point of the actuator perpendicular to and in the direction of printing, are determined. These values are used to determine the skewing and bulging of the actuators.

Secondly, the displacement point clouds are then filtered on the middle thickened part of the actuator (a circle with radius $14.5 \mathrm{~mm}$, as shown in Figure 2). All further conclusions and analysis on skewing, tilting, specific displacement and bulging are made on this part of the actuator.

Subsequently, a mean displacement of the thickened part with a standard deviation will be determined. The standard deviation is a measure of the accuracy of the scanning method and the skewing. However, it also reflects the misalignment of the surface roughness between the reference point cloud and the calculated point clouds.

\subsection{Microscopy}

As the membrane thickness is one of the main geometrical parameters which determine the displacement ratio and stroke, it has to be further investigated. This is measured by cutting the prototypes open, grinding and polishing them, and subsequently inspecting the sectioned pieces under the microscope as can be seen in Figure 4a-c. Each cross-section is pictured and analysed using image recognition software. This software is developed in Matlab. It calculates the porosity, thickness and roughness on a pixel count basis. Each pixel has a height and width of $1.8 \mu \mathrm{m}$. Pores smaller than a total of 10 pixels are not taken into account, as they will not influence mechanical properties of the membranes significantly. It is assumed that the porosity of a cross-section can be extrapolated to the porosity of the membrane geometry.

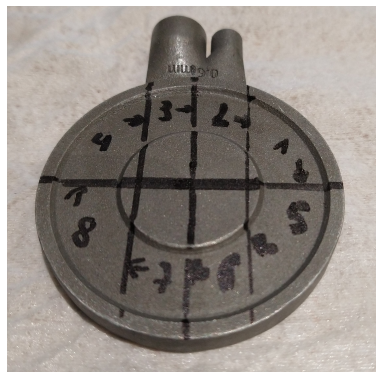

(a)

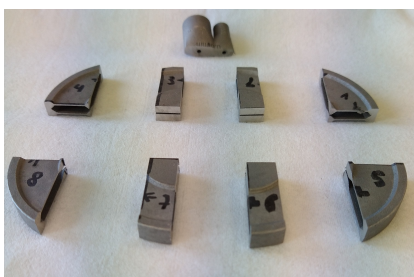

(b)

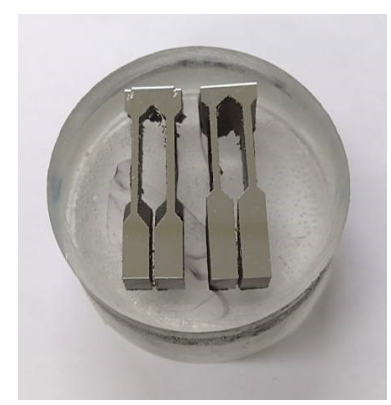

(c)

Figure 4. The prototypes are prepared for microscopy by cutting them in eight pieces (plus threaded hydraulic connection, which is discarded), putting them into epoxy embedding material to subsequently grind and polish them. (a) Top view of the of the prototype before cutting. The parts are marked and numbered before cutting. (b) Top view of the eight cut pieces (and the threaded connections) after cutting. (c) View of one of the cut pieces in the embedding material after grinding and polishing, ready for the microscope. 


\section{Results}

Colour maps of the specific displacement of the membrane of each actuator can be seen in Figure 5. Below and at the side of each colour map, the specific displacement of a $1 \mathrm{~mm}$ wide section, as indicated with fine black lines, in the print direction ( $y$-axis, vertically) and perpendicular to the print direction ( $x$-axis, horizontally) is plotted on the charts. These two cross-sectional views reveal a correlation between skewing of the membrane and the print direction of the LPBF process, especially with the thinner membranes (Figure $5 d, f$ ).

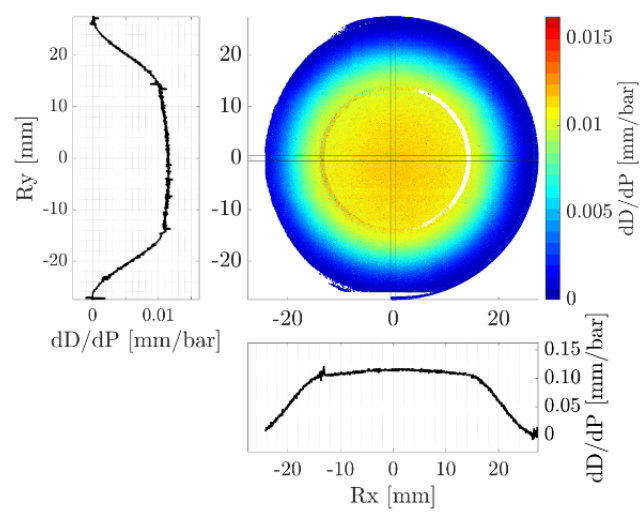

(a) Membrane $1 \mathrm{~A}$ at 6.00 bar

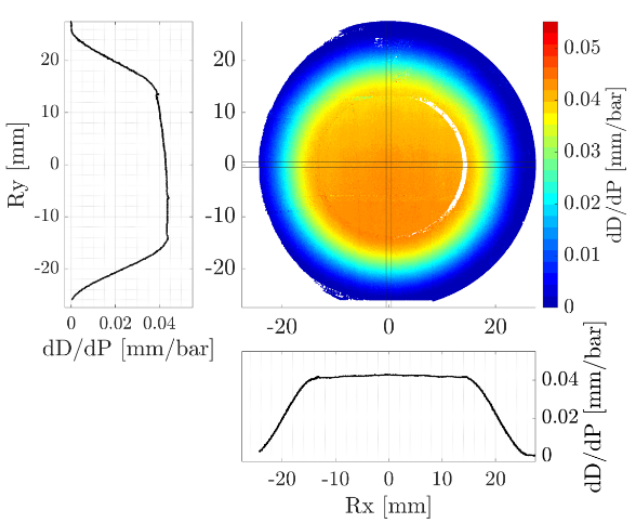

(c) Membrane 2A at 2.25 bar

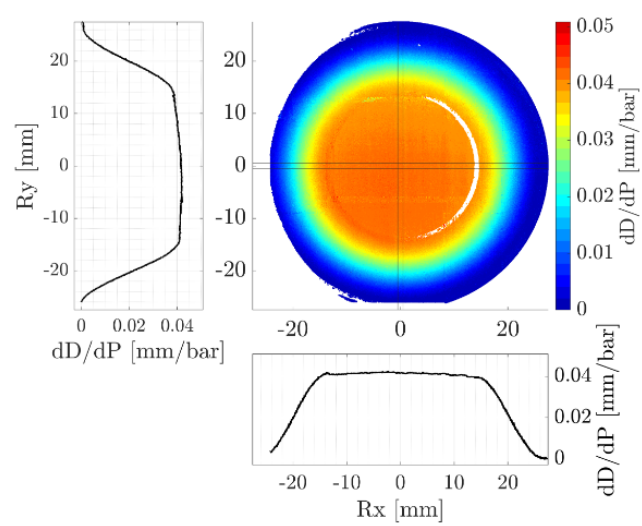

(e) Membrane 3A at 2.25 bar

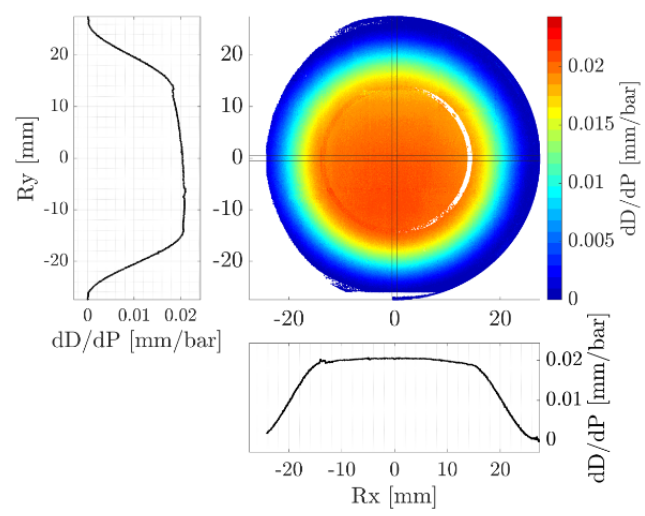

(b) Membrane 1B at 6.00 bar

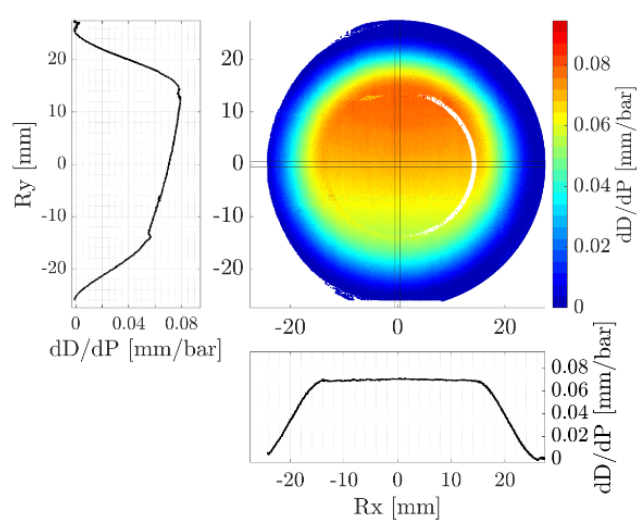

(d) Membrane 2B at 1.25 bar

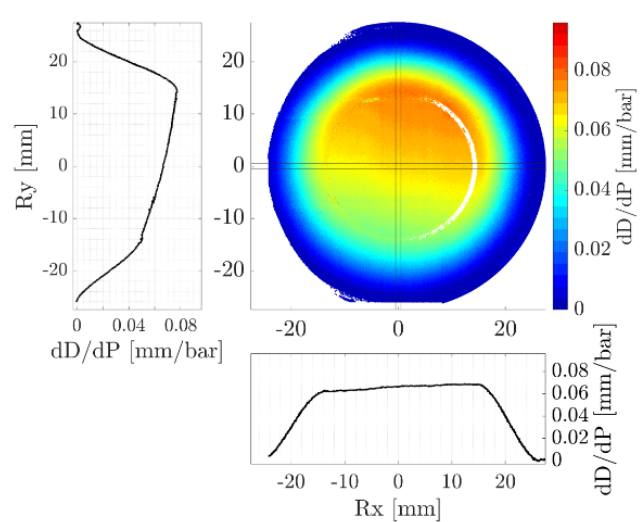

(f) Membrane 3B at 1.25 bar

Figure 5. Colour maps of the specific displacements at a certain pressure of the membranes. Bellow and at the side of each colour map the average specific displacement of a $1 \mathrm{~mm}$ wide section, as indicated with fine black lines, in the print direction ( $y$-axis, vertically) and perpendicular to the print direction ( $x$-axis, horizontally) is displayed. This section gives a clear view of the skewing of the thinnest prototypes (Figure $5 \mathrm{~d}, \mathrm{f}$ ) in the direction of printing. 
A linear correlation between the applied pressure on the membrane surface and displacement is expected, as the positioner is based on the flexibility of the material within the elastic domain, which follows Hooke's law. The mean displacements can be used to calculate the specific displacement $\frac{d D}{d P}\left[\mu \mathrm{mbar}^{-1}\right]$ with a first-order linear fit. Each linear fit has an $R^{2}>0.99$, which confirms the linearity between the applied pressure and displacement within the limits of the tested pressures.

Microscope images (Figure 6a) are analysed with a feature recognition program (Figure 6b). In red are all the detected pores, in green, the found edges, in white, the membrane and the background is black.

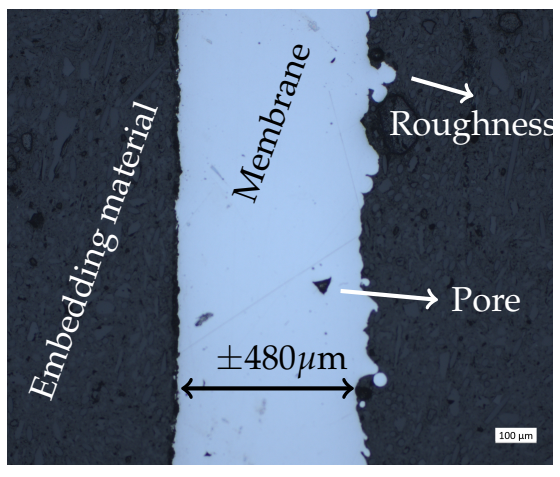

(a)

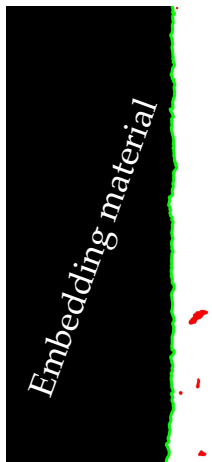

(b)

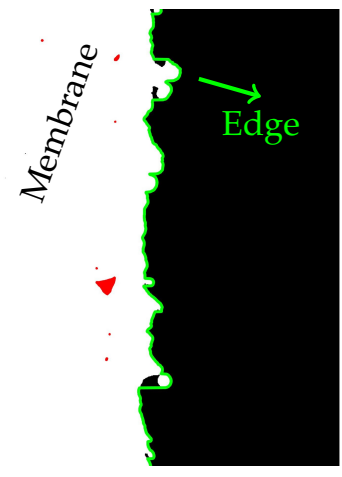

b)

Figure 6. In figure (a), an image of the microscope is shown, the embedding material in black and the membrane in grey. In figure (b), the analysed data is visualised, in red all the detected pores, in green the detected edges, in white the membrane section and the background is black. The code calculates the roughness, the porosity (pore area compared to total membrane area) and the thickness of the membrane on a pixel count basis.

About seventy pictures (depending on the thickness of the membrane) are taken for each membrane to determine porosity, roughness and thickness. Porosity is a measure of the pore area compared to the total area of the cross-section. The following could be concluded from the analysis: the average thickness of each membrane is thinner than designed, whereas the maximum thickness, and thus the thickness that is measured using conventional tactile measuring methods, is close to the design thickness. The roughness on the inside of the membrane (the pressurised side) is higher than the outside of the membrane due to sandblasting surface treatment after printing. The roughness of the outside of the membranes varied between $60.4 \mu \mathrm{m}$ up to $118.9 \mu \mathrm{m}$, as can be seen in Table 2 . Most pores are found about $100 \mu \mathrm{m}$ from the edge of the membrane, most likely due to a non-optimized printing pattern in which the gap between fill and contours print vectors is too large [12]. Only a few pores are significantly large to impact the elastic properties of the membrane, whereas most pore's diameters are smaller than $15 \mu \mathrm{m}$. The membrane with the highest porosity is the second thickest membrane (3A) with a porosity area of $0.27 \%$ (see Table 2). Which is lower than the typical LBPF porosity of up to $0.5 \%$ [13].

Skewing of the membrane tends to be mainly in the direction of printing. This is especially notable in the thinner membrane prototypes, as can be seen on Figure $5 \mathrm{~d}$,f. Microscopy analysis of the prototypes did not reveal a significant difference in thickness, porosity or roughness in the direction of printing. The skewing might be the result of internal stresses caused by the heat of the manufacturing process, which has a bigger impact on thin membranes, as they transport heat less effectively [14]. Bulging of the thickened part of the membrane can be seen for the thickest membrane (Figure 5a), due to the high stiffness of the membrane itself and thus the higher required pressures. 
Table 2. The results of the microscopy image analysis and the scanning analysis; design thickness, average thickness, Standard deviation on the thickness $\left(\operatorname{STD}_{\text {Thickness }}\right)$, specific displacement $\left(\left|\frac{d D}{d P}\right|\right)$, surface roughness and porosity for each investigated membrane. Each membrane has a lower average thickness as per design due to the surface roughness. The thinnest membranes.

\begin{tabular}{llcccccc}
\hline \multirow{2}{*}{ Property } & \multirow{2}{*}{ Unit } & \multicolumn{7}{c}{ Prototype } \\
\cline { 3 - 7 } & & 1A & 1B & 2A & 3A & 2B & 3B \\
\hline Design thickness & {$[\mu \mathrm{m}]$} & 1200 & 1000 & 800 & 800 & 600 & 600 \\
Average Thickness & {$[\mu \mathrm{m}]$} & 1058.4 & 861.2 & 673.9 & 672.6 & 477.2 & 480.5 \\
STD $_{\text {Thickness }}$ a & {$[\mu \mathrm{m}]$} & 21.9 & 24.7 & 23.3 & 23.7 & 24.7 & 27.4 \\
Specific displacement $_{\text {Skewing }}\left[\mu \mathrm{mbar}^{-1}\right]$ & 11.1 & 19.9 & 42.0 & 40.5 & 68.1 & 62.3 \\
Surface roughness & $\mu \mathrm{mar}^{-1}$ & $<3$ & $<3$ & $<3$ & $<3$ & 25.1 & 28.3 \\
Porosity & {$[\mu \mathrm{m}]$} & 108.3 & 118.9 & 60.4 & 85.2 & 61.5 & 90.4 \\
Performance & {$[\%]$} & 0.20 & 0.27 & 0.1 & 0.08 & 0.07 & 0.07 \\
\hline
\end{tabular}

${ }^{a}$ The standard deviation on the thickness is due to the differences in thickness in different microscopic images and incorporates the surface roughness. ${ }^{\mathrm{b}}$ Membranes $2 \mathrm{~A}$ and $3 \mathrm{~A}$ were deemed best because of the high specific displacement, low skewing and low flexing of the thickened part.

The specific displacement in $\mu \mathrm{mbar}^{-1}$ as function of the membrane thickness is shown in Table 2. One can immediately note the big range between the minimum thickness and the maximum thickness of each membrane. However, these values are cross-section measurements of a 3D membrane, thus these extreme minimum values are most likely local indents, with only a minor impact on overall elastic properties. Thicker membranes appear to have larger indents and protrusions.

The standard deviation on the mean displacement (Table 2) is mainly due to the roughness of the membrane, no significant difference in thickness on different locations of the membranes is found. The standard deviation on the thickness is slightly larger in thinner membranes than thicker membranes, suggesting a higher roughness of the thinner membranes.

The measured specific displacements are larger than the finite element simulation predicted, even when taking into account the difference between the measured thickness and the design thickness. This can be seen in Figure 7a, where the grey dotted line shows the FES results, the dots and squares depict the different tested membranes and prototypes. The minimum and maximum thickness of each membrane is connected with coloured dotted lines, the standard deviation is depicted with an error bar. A multitude of collaborative reasons may be the cause of this shift. First of all, will the porosity affect the effective E-modulus of the membrane, however in the worst-case scenario this would decrease the Young's modulus (and thus increase the expansion ratio) by about $1 \%$ [15]. Secondly, the roughness of the membranes is significantly high, thus decreasing the effective thickness of the membrane.

In order to improve simulations, thus to improve the predictability of the specific displacement of the membranes, a correction should be incorporated in the FE simulations. Ideally, this correction would incorporate a surface roughness model and a porous model in the existing CAD model. However, in the interest of fast prototyping, a less arduous solution is sought. Two solutions without the need for invasive remodelling of the CAD models are; a thickness correction and a Young's modulus correction. The thickness correction would consist of a FE simulation with an adapted thickness, which would be used for simulations and added/subtracted to the CAD files to manufacture the actuator. A Young's modulus correction is even less invasive and would simply consist of an adapted Young's modulus for the simulations. 


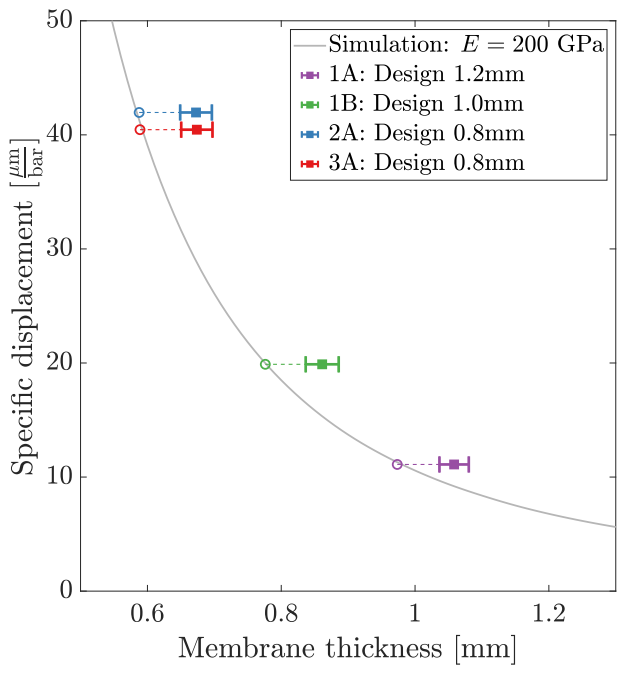

(a) Thickness correction

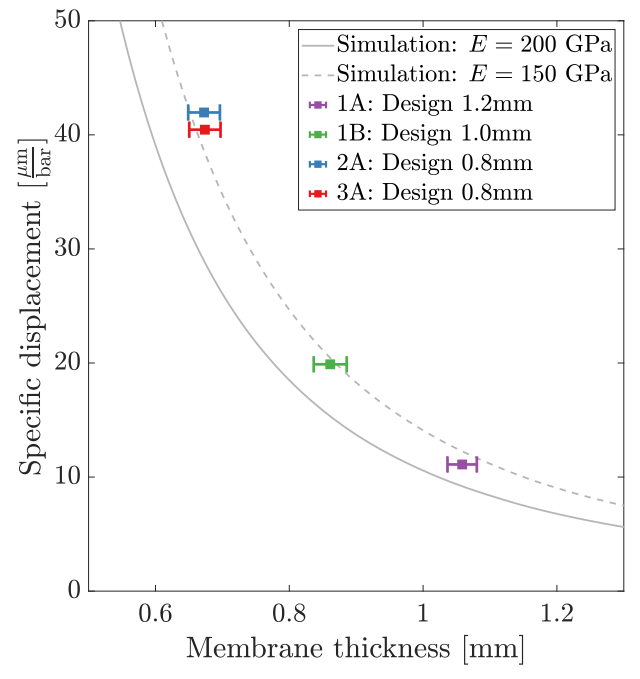

(b) Youngs modulus correction

Figure 7. The specific displacement of each membrane thicker then $0.6 \mathrm{~mm}$ in function of the membrane thickness is depicted on both charts. On the left pane, a thickness correction method is shown, which consists of a static thickness which is subtracted from the membrane thickness. The gray line depicts the FES, and the circles depict a thickness correction of $85 \mu \mathrm{m}$. On the right pane a Young's modulus correction is depicted, which consists of a reduction of the Young's modulus used in the simulation. The gray full line depicts again the FES with a Young's modulus of $200 \mathrm{GPa}$, whereas the dotted line depicts a FES with a Young's modulus of $150 \mathrm{GPa}$. The thickness correction appears to be slightly more accurate in predicting the specific displacement than the Young's modulus correction; however, the Young's modulus correction is easier to implement.

A thickness correction of $85 \mu \mathrm{m}$ on the average thickness of the membranes resulted in an accurate model for the expansion ratio of the membranes (see Figure 7a). Although the Young's modulus correction can be accurate an accurate compensation in FES of ceramics, as done in the work by $[15,16]$, however, it proved to be less accurate in predicting the effect of the surface roughness in metal printed parts (see Figure 7b).

\section{Discussion}

Due to the surface roughness, the effective membrane thicknesses tended to be thinner than designed by $119.5 \mu \mathrm{m}$ to $141.6 \mu \mathrm{m}$. Although simulations predicted that thinner membranes would offer large strokes and large specific expansions, membranes thinner than $0.6 \mu \mathrm{m}$ are deemed unfavourable for making actuators with current LPBF technologies.

First of all, the skewing of the membrane during actuation is too large, in membrane 3B (see Figure $5 \mathrm{f}$ ) a skewing of $30 \mathrm{~m} \mathrm{bar}^{-1}$ is measured, on an average expansion ratio of $62.3 \mu \mathrm{mbar}^{-1}$.

Secondly, because of the relatively high roughness of the membranes, the behaviour of thin membranes becomes difficult to predict, with high skewing in the direction of the print.

Lastly, because of the small strokes of the membrane actuators and the relatively high surface roughness LPBF parts have, machining of the thickened part of the membrane actuator would be required. Thin membranes are too flexible, which makes further machining more difficult or impossible.

Thick membranes of $1.0 \mathrm{~mm}$ and higher have little skewing, however they achieve a relatively low specific displacement of $11.1 \mu \mathrm{mbar}^{-1}$. Furthermore, is the thickened part in the middle of the membrane bulging due to the high required pressures. Membrane thickness of $0.6 \mathrm{~mm}$ up to $1.0 \mathrm{~mm}$ achieved favourable specific displacements, up to $42.0 \mathrm{um} \mathrm{bar}^{-1}$, with little to no skewing or bulging of the thickened part. 
Membranes of design thickness $0.8 \mathrm{~mm}, 1 \mathrm{~mm}$ and $1.2 \mathrm{~mm}$ tended to have a larger expansion ratio than the FES with their average thickness suggested (as can be seen in Tables 1 and 2). A combination of roughness and porosity of the membrane is proposed as an explanation for this offset. A Young's modulus correction improved the FES results, but a thickness correction during FES proved to be more accurate.

\section{Conclusions}

The basic prototype design allowed for in-depth research in the behaviour of the LPBF manufactured steel membranes. First of all, a minimum membrane thickness of $0.6 \mathrm{~mm}$ was determined using current LPBF technology. This minimum thickness is set in order to avoid skewing of the membrane surface. In addition, it is important to take into account that the design thickness of the membranes can differ significantly from the actual thickness. This is mainly due to the high surface roughness of the LPBF printing process. Secondly, a deviation from the simulated specific displacement was measured. The porosity of the membranes was relatively low in comparison with other 316L LBPF parts, thus it is assumed that the main reason of the deviation is a combination fo the surface roughness of the membranes and a loss of the effective Young's modulus due to the thickness of the membranes.

Two compensation strategies are suggested to improve the predictability of the specific displacement. In the future design process of high stiffness, compact micro positioning devices manufactured using LPBF, based on intentional elastic deformation, these should be taken into account. The first one being a thickness correction. This thickness should be subtracted from the membrane thickness when running the FES, for a more accurate prediction of the specific displacement of the actuators. Secondly the Young's modulus could be adapted from the given Young's modulus of the supplier, to a lowered effective Young's modulus.

Because of the high strength of 316L stainless steel and the geometrical manufacturing possibilities of LPBF, micro positioning devices based on the membrane type design suggested in this communication, could be used in applications that demand an accurate, rigid and compact micro range actuator. In a static actuating environment this would mean that the actuator can deliver high actuating forces. This force is in proportion to the pressure and membrane thickness. Whereas, in an dynamic environment, the high rigidity would allow the actuators, the withstand high dynamic loads with a minimum displacement and/or damage to the actuator. This without the need of complex active dynamic compensation strategies. However, the application of these kind of actuators remains a niche, as the stroke is limited by the yield strength of the actuator, which in the case of these prototypes is only several hundred micrometer.

Taking into account the minimum membrane thickness and suggested compensation strategies, a doorway opens to more complexly shaped micro positioning devices using induced elastic deformation.

Author Contributions: Writing and original draft preparation, methodology, software, validation and formal analysis, F.F. Conceptualization, F.F. and B.V. Project administration, supervision, B.V. and S.B. Resources, funding acquisition, F.F., B.V. and S.B. All authors have read and agreed to the published version of the manuscript.

Funding: Flanders Innovation \& Entrepreneurship Agency (VLAIO) is gratefully acknowledged for its support within the Baekeland mandate (VLAOO14).

Institutional Review Board Statement: Not applicable.

Informed Consent Statement: Not applicable.

Conflicts of Interest: The authors declare no conflict of interest. 


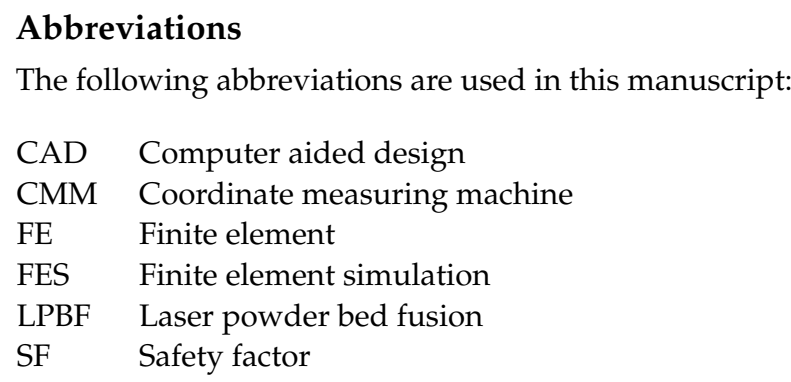

\section{References}

1. Gorissen, B.; Vincentie, W.; Al-Bender, F.; Reynaerts, D.; Volder, M.D. Modeling and bonding-free fabrication of flexible fluidic microactuators with a bending motion. J. Micromech. Microeng. 2013, 23, 045012. [CrossRef]

2. Zolfagharian, A.; Mahmud, M.A.P.; Gharaie, S.; Bodaghi, M.; Kouzani, A.Z.; Kaynak, A. 3D/4D-printed bending-type soft pneumatic actuators: Fabrication, modelling, and control. Virtual Phys. Prototyp. 2020, 15, 373-402. [CrossRef]

3. Kang, H.W.; Lee, I.H.; Cho, D.W. Development of a micro-bellows actuator using micro-stereolithography technology. Microelectron. Eng. 2006, 83, 1201-1204. [CrossRef]

4. Fateri, M.; Carneiro, J.F.; Frick, A.; Pinto, J.B.; Gomes de Almeida, F. Additive Manufacturing of Flexible Material for Pneumatic Actuators Application. Actuators 2021, 10, 161. [CrossRef]

5. Maruthavanan, D.; Seibel, A.; Schlattmann, J. Fluid-Structure Interaction Modelling of a Soft Pneumatic Actuator. Actuators 2021, 10, 163. [CrossRef]

6. Blanes, C.; Mellado, M.; Beltran, P. Novel Additive Manufacturing Pneumatic Actuators and Mechanisms for Food Handling Grippers. Actuators 2014, 3, 205-225. [CrossRef]

7. De Volder, M.; Reynaerts, D. Pneumatic and hydraulic micro-Actuators: A review. J. Micromech. Microeng. 2010, $20,043001$. [CrossRef]

8. Delgado, J.; Ciurana, J.; Serenó, L. Comparison of forming manufacturing processes and selective laser melting technology based on the mechanical properties of products. Virtual Phys. Prototyp. 2011, 6, 167-178. [CrossRef]

9. Deev, A.; Kuznetcov, P.; Petrov, S. Anisotropy of Mechanical Properties and its Correlation with the Structure of the Stainless Steel 316L Produced by the SLM Method. Phys. Procedia 2016, 83, 789-796. [CrossRef]

10. Roach, A.M.; White, B.C.; Garland, A.; Jared, B.H.; Carroll, J.D.; Boyce, B.L. Size-dependent stochastic tensile properties in additively manufactured 316L stainless steel. Addit. Manuf. 2020, 32, 101090. [CrossRef]

11. Chahal, V.; Taylor, R.M. A review of geometric sensitivities in laser metal 3D printing. Virtual Phys. Prototyp. 2020, 15, $227-241$. [CrossRef]

12. Andreau, O.; Pessard, E.; Koutiri, I.; Peyre, P.; Saintier, N. Influence of the position and size of various deterministic defects on the high cycle fatigue resistance of a 316L steel manufactured by laser powder bed fusion. Int. J. Fatigue 2021, 143, 105930. [CrossRef]

13. Lavery, N.; Cherry, J.; Mehmood, S.; Davies, H.; Girling, B.; Sackett, E.; Brown, S.; Sienz, J. Effects of hot isostatic pressing on the elastic modulus and tensile properties of 316L parts made by powder bed laser fusion. Mater. Sci. Eng. A 2017, 693, 186-213. [CrossRef]

14. Yadroitsev, I.; Yadroitsava, I. Evaluation of residual stress in stainless steel 316L and Ti6Al4V samples produced by selective laser melting. Virtual Phys. Prototyp. 2015, 10, 67-76. [CrossRef]

15. Roberts, A.P.; Garboczi, E.J. Elastic Properties of Model Porous Ceramics. J. Am. Ceram. Soc. 2000, 83, 3041-3048. [CrossRef]

16. Knudsen, F.P. Effect of Porosity on Young's Modulus of Alumina. J. Am. Ceram. Soc. 1962, 45, 94-95. [CrossRef] 